\title{
Evaluation of Fast-Time Wake Vortex Prediction Models
}

\author{
Fred H. Proctor ${ }^{*}$ and David W. Hamilton ${ }^{\dagger}$ \\ NASA Langley Research Center, Hampton, Virginia, 23681
}

\begin{abstract}
Current fast-time wake models are reviewed and three basic types are defined. Predictions from several of the fast-time models are compared. Previous statistical evaluations of the APA-Sarpkaya and D2P fast-time models are discussed. Root Mean Square errors between fast-time model predictions and Lidar wake measurements are examined for a $24 \mathrm{hr}$ period at Denver International Airport. Shortcomings in current methodology for evaluating wake errors are also discussed.
\end{abstract}

\section{Introduction}

$\mathrm{V}$ ORTICITY is generated as a consequence of aerodynamic lift from the wings, flaps, tail and body of the aircraft. Complete roll-up of this vorticity into a pair of counter-rotating trailing vortices usually occurs within about 20 wing spans downstream of the aircraft ${ }^{1}$. The lateral spacing between the rolled-up vortices is initially about $78 \%$ of the aircraft's wing span, but may vary several percent due to non-elliptical wing loading and aircraft configuration. The initial strength for each of the rolled-up vortices (represented by its circulation) is proportional to the generating aircraft's weight and inversely proportional to air density, airspeed, and wing span. Large aircraft tend to produce stronger vortices than smaller aircraft, since the heavier weight of large aircraft more than offsets their larger wingspan. Wake vortices descend downward due to mutual induction of the vortex pair, as they are transported with the ambient wind. Meteorology greatly affects the transport and decay of wake vortices. Interaction with the ground adds further complexity to the evolution of wake vortices. Wake vortices that descend into ground effect (IGE) begin to separate laterally and decay more quickly than those that remain out of ground effect (OGE). Lifetimes of wake vortices range from about 20 seconds to several minutes, depending upon the generating aircraft, proximity to the ground, and meteorological conditions. Aircraft encounters with wake vortices can be unsafe due to the potential for loss of control. Maintaining ample separation between aircraft can overcome this danger, but overly conservative separations may reduce capacity and increase delays, especially during periods of heavy traffic.

Current aircraft separation standards are conservatively based on aircraft weight categories to avoid unsafe wake encounters. Additional system capacity may be gained, while maintaining the current levels of safety, by including factors that affect wake behavior. For example, the presence of significant crosswinds or turbulence may limit the residence of wake vortices within a flight corridor and create the potential for less restrictive aircraft separations. In order to realize this benefit, fast-time wake prediction models are needed that reliably predict wake characteristics based on atmospheric and aircraft inputs. Other applications of fast-time models include 1) guidance for wake separation standards for new aircraft, 2) optimizing separation standards for existing aircraft, 3) guidance for setting vertical separation standards during cruise, 4) accident reconstructions, and 5) testing of new concepts for safe increases in airport capacity for both single and multiple runway configurations. Fast-time prediction models could also be used in the cockpit -- with input from airborne sensors and traffic position broadcast -- to provide real-time wake hazard information to the pilot. ${ }^{23,4}$

Fast-time models are semi-empirical, and are designed to predict wake vortex information behind a generating

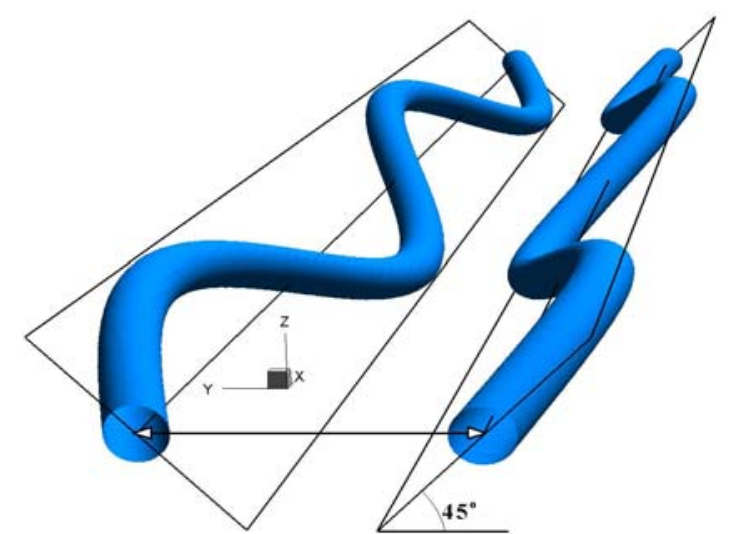

Figure 1. Schematic of vortex pair undergoing sinusoidal instability.

\footnotetext{
* Research Meteorologist, Crew Systems \& Aviation Operations Branch, MS 156A, AIAA Senior Member.

† Senior Scientist, Current Affiliation: WeatherPredict Consulting Inc, Raleigh NC.
} 
aircraft. They are developed using theoretical concepts and often calibrated with data obtained from field measurements, laboratory studies, or numerical simulations. They rely upon inputs representing the generating aircraft (such as weight, airspeed, wingspan, lateral position, and altitude) and atmospheric environment (such as ambient winds, turbulence, and temperature lapse rate). Other input may include the type of aircraft (e.g., B-747400) and phase of flight (departure, descent, or cruise), since different aircraft types have different wing loadings that can also vary depending upon phase of flight.

Fast-time models should provide solutions in a fraction of a second, and hence are able to perform in a real-time operational system, such as the dynamic spacing algorithms envisioned by the Aircraft Vortex Spacing System (AVOSS), ${ }^{5,6,7}$ or the Wake Vortex Advisory System (WakeVAS) ${ }^{8,9}$. Fast-time models are based on a number of assumptions which limit their accuracy and range of application. Fast-time models do not provide the wake-vortex flow field details found in Large Eddy Simulation (LES) models such as can be provided with the Terminal Area Simulation System (TASS) ${ }^{10,11}$. Fast-time models can not directly predict the meandering vortex paths resulting from atmospheric turbulence, sinusoidal displacements (Fig. 1), or vortex breakup into vortex rings resulting from Crow instability ${ }^{12}$. However, they should account for the mean vortex position and the effects of Crow instability on circulation decay, as well as the uncertainty caused by these interactions.

Fast time models can be divided into three types:

- Deterministic fast-time models - predict the discrete vortex trajectories and circulation vs time, e.g., Refs. $13,14,15,16,17 ; 18$

- Wake zone fast-time models - predict bounded area where wake vortex may reside; may be based on worst case conditions, ${ }^{19}$ or could be a deterministic fast time model with bound predictions that are based upon anticipated environmental and aircraft uncertainties; ${ }^{20}$

- Probabilistic fast-time models - predict the probability that a wake vortex will be a particular distance from the flight path and the probability that the wake vortex has weakened below a specified strength. Probabilistic models can be entirely data driven or use either a single or an ensemble of deterministic models as its basis.

Deterministic fast-time models should include as much physics as possible to allow robustness and reliability of their solutions. They should include formulations for vortex decay based on atmospheric turbulence and stratification. An acceptable deterministic model can provide the basis for both wake zone and probabilistic fasttime models. The achievement of the latter is an important goal since such models would be highly useful in safety vs benefit studies and flight-spacing optimizations. Validation of the models with aircraft wake measurements is an essential step before applying them to safety-critical applications.

Many of the fast-time wake prediction models use a parameter representing atmospheric turbulence as input. Eddy dissipation rate (EDR) is recommended over velocity variance or Turbulence Kinetic Energy (TKE) for reasons that include the following. Atmospheric turbulence has a continuous spectrum and is rarely represented by a discrete gust. Scales that affect wake vortex decay are on the order of the aircraft wingspan and are usually well within the inertial subrange. In contrast, scales of turbulence that primarily contribute to TKE and velocity variance can be as large as $1-3 \mathrm{~km}$ and depend upon the depth of the atmospheric boundary layer ${ }^{21}$. The time scales for the larger eddies are much longer than the lifetime of wake vortices.

\section{Candidate Models}

An objective of NASA's Airportal Project of the Airspace Systems Program is to assess the accuracy of available deterministic fast-time wake prediction models and make improvements where needed. Candidate models include the AVOSS Prediction Algorithm (APA) ${ }^{15,22}$ with the OGE transport and decay computed using either Sarpkaya $^{23}$, the TASS Driven Algorithm for Wake Prediction (TDAWP) ${ }^{24}$, or the Deterministic 2-Phase (D2P) ${ }^{25}$ model (as re-coded from literature).

The APA model predicts wake vortex trajectories and circulation within a plane perpendicular to the path of the generating aircraft. Atmospheric inputs include vertical profiles of the ambient crosswind, temperature, and turbulence intensity (represented by EDR). The initial wake is represented as two vortices whose initial strength and position are dependent upon input conditions. If the wake is out of ground effect, the APA model utilizes a decay and transport model, such as developed by Sarpkaya ${ }^{23}$. For in ground effect, APA utilizes image vortices to represent the effect of an impenetrable ground, and the introduction of secondary vortices that cause wake vortex rebound. The method for vortex decay while in ground effect depends on the version of APA. In version 3.1.1, the same vortex decay rate that is computed for OGE is assumed to persist throughout the IGE phase. In version 3.2, IGE decay is computed with a formula derived from a TASS LES study ${ }^{26}$. Unpublished statistical evaluations 
conducted by NorthWest Research Associates (NWRA) have shown smaller errors between APA 3.2 and field measurements than with version 3.1.1.

The latest APA version, Suite 4.0 , is similar to APA 3.2, but allows the option of using Sarpkaya, TDAWP, or D2P as the OGE module (Fig. 2). The source code for this update was recently completed by NWRA under NASA contract NNL06AC78T.

The Sarpkaya OGE model ${ }^{15,23}$ assumes two parallel vortices that decay and descend at equivalent rates. The model has one prognostic equation governing circulation and contains terms for decay due tor ambient stratification and turbulence. Vortex descent is determined by using the predicted circulation and a diagnostic relationship for vortex separation. The model's empirical constants were adjusted using data collected

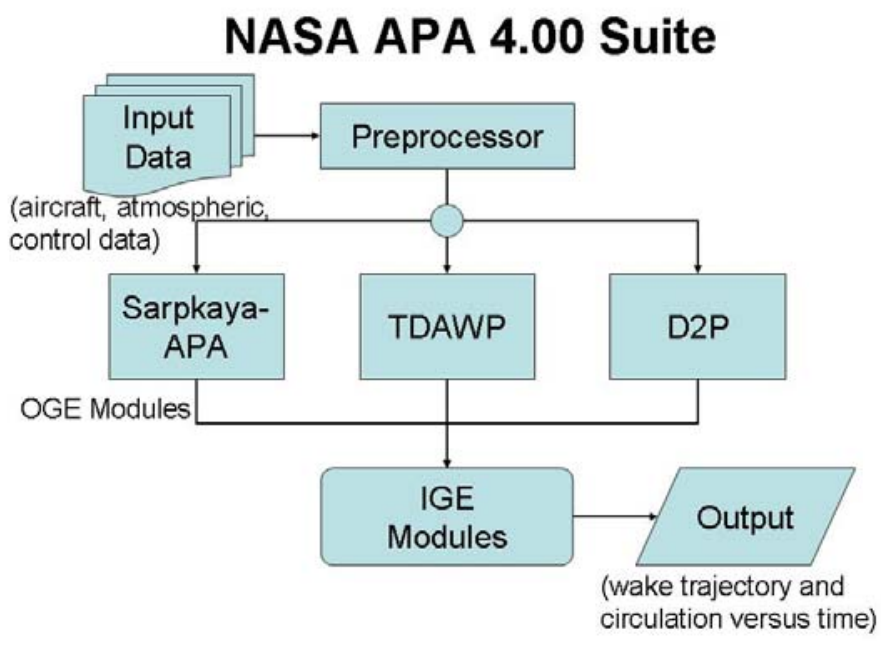

Figure 2. Architecture of APA 4.0 Suite.

during NASA's Memphis ${ }^{27}$ and Dallas-Fort Worth ${ }^{28}$ field studies. In these data campaigns, measurements of aircraft wake vortices were acquired with Continuous-Wave (CW) Lidar and meteorological profiles were obtained from a composite of sensors located on the airport's property.

D2P is the deterministic portion of the Probabilistic 2-Phase (P2P) model ${ }^{\ddagger}$ and is described in Refs. 16, 20, and 25. As in Sarpkaya's model, two parallel vortices are assumed to decay and descend at equivalent rates. However, in D2P circulation decay is governed by an algebraic relationship representing the decay of a single potential vortex. The algebraic relationship incorporates effects from stratification and ambient turbulence as guided by LES results ${ }^{29}$. Similarly, the vortex descent rate is empirically diagnosed from the circulation. The predicted 5-15 m average circulation $^{\S}$ in D2P is characterized by two-phases of decay: the first being a "diffusion" phase followed by a more rapid rate of decay as indicated in LES experiments.

The TDAWP model has separate prognostic equations for vortex descent rate and 5-15 $\mathrm{m}$ average circulation, and it applies these separately to both the port and starboard vortices ${ }^{24}$. The formulation is driven by parametric studies from LES using TASS ${ }^{30,31}$. Unlike the previously described OGE models, the TDAWP formulation also includes the effects of crosswind shear on vortex descent rate, thus allowing the prediction of vortex tilt and the change in lateral separation due to crosswind. Currently, the effects of crosswind shear on circulation decay are not treated in TDAWP, nor in APA, Sarpkaya, and D2P.

\section{Comparison of Results from Fast-time Models}

In this section several experiments are conducted in order to illustrate some of differences and similarities between the results from the three OGE models in APA 4.0 Suite.

In the first set of experiments, the APA 4.0 Suite is run for Sarpkaya, TDAWP and D2P, with three theoretical environments. For reference, APA 4.0 with Sarpkaya is identical to APA 3.2. The three cases assume no ambient crosswind, and represent an environment with either 1) low turbulence and neutral stratification, 2) strong turbulence and neutral stratification, or 3) low-turbulence and isothermal (stable) stratification. The low turbulence cases assume a constant environmental EDR of $10^{-4} \mathrm{~m}^{2} \mathrm{~s}^{-3}$. The strong turbulence case assumes an EDR of $10^{-2} \mathrm{~m}^{2} \mathrm{~s}^{-3}$ (representative of the upper range of turbulence within the atmospheric boundary layer). In all of the cases, an initial circulation of $575 \mathrm{~m}^{2} \mathrm{~s}^{-1}$, initial vortex separation of $50 \mathrm{~m}$, and generation height of $300 \mathrm{~m}$ are assumed. Model predictions for altitude and circulation are shown in Figs. 3 and 4, respectively.

\footnotetext{
‡ According to our definition, P2P is a "wake zone fast-time model” rather than a "fast-time probabilistic model” as referred to by its authors.

$\S^{\S}$ The 5-15 $\mathrm{m}$ average circulation represents a radial average of circulation between a vortex radius of 5 and $15 \mathrm{~m}$.
} 

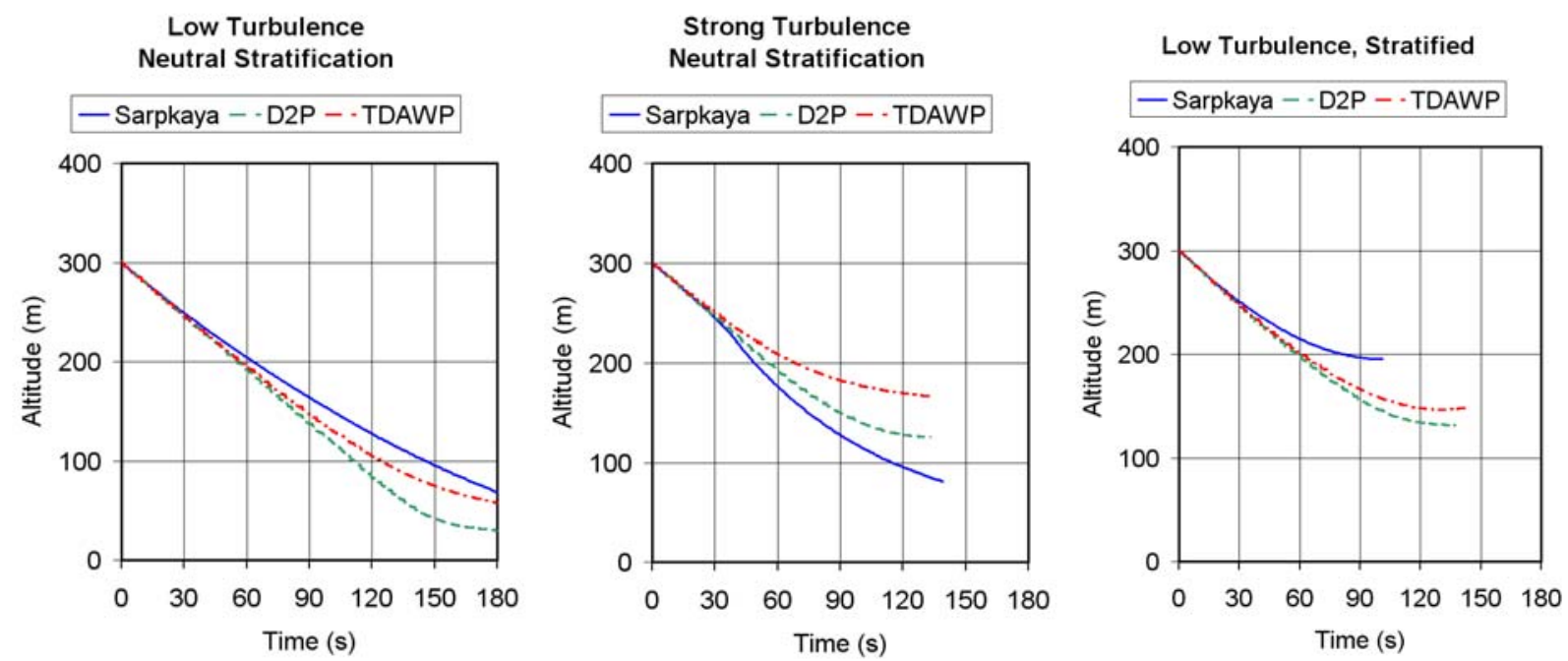

Figure 3. Altitude vs time comparison between OGE models.

Altitude predictions (Fig. 3) are very similar for the three models during the first half of the wake lifetime. The greatest differences occur for the environment with strong turbulence. For this environment, Sarpkaya predicts the wake vortices descend to a greater depth than TDAWP, with D2P somewhere in between. For the low-turbulence and neutral stratification case, Sarpkaya's descent is slightly weaker than TDAWP, with D2P having the greatest descent. For the stratified condition, TDAWP and D2P descend at nearly similar rates, but Sarpkaya's descent is arrested at a noticeably higher altitude.

The predictions for circulation in Fig. 4 show large differences between Sarpkaya and the other two models. The prediction of circulation decay from Sarpkaya for neutral stratification has a convex shape with an initially-rapid decay followed by a slower rate of decay at later times. In comparison, both the D2P and TDAWP predictions show an initially slow rate of decay followed by a more rapid decline in circulation. For near neutral stratification, Sarpkaya predicts weaker vortices at vortex mid-lifetime, but a longer vortex lifetime. For the stratified case, the Sarpkaya model shows a sustained rapid decay with a shorter vortex lifetime than the other two models. The rate of change of circulation predicted by D2P in Fig. 4 shows a two-phase decay, with the first phase appearing linear. The predictions from TDAWP are similar, but have a smoother change in slope and have a slightly stronger circulation than D2P. The vortex lifetime predictions with TDAWP are generally longer than with D2P.

In order to illustrate differences in APA predictions in the presence of nonlinear vertical shear of crosswind, the Idaho Falls B-757 Run 9 benchmark case is chosen. For this case, wake vortex measurements were made with
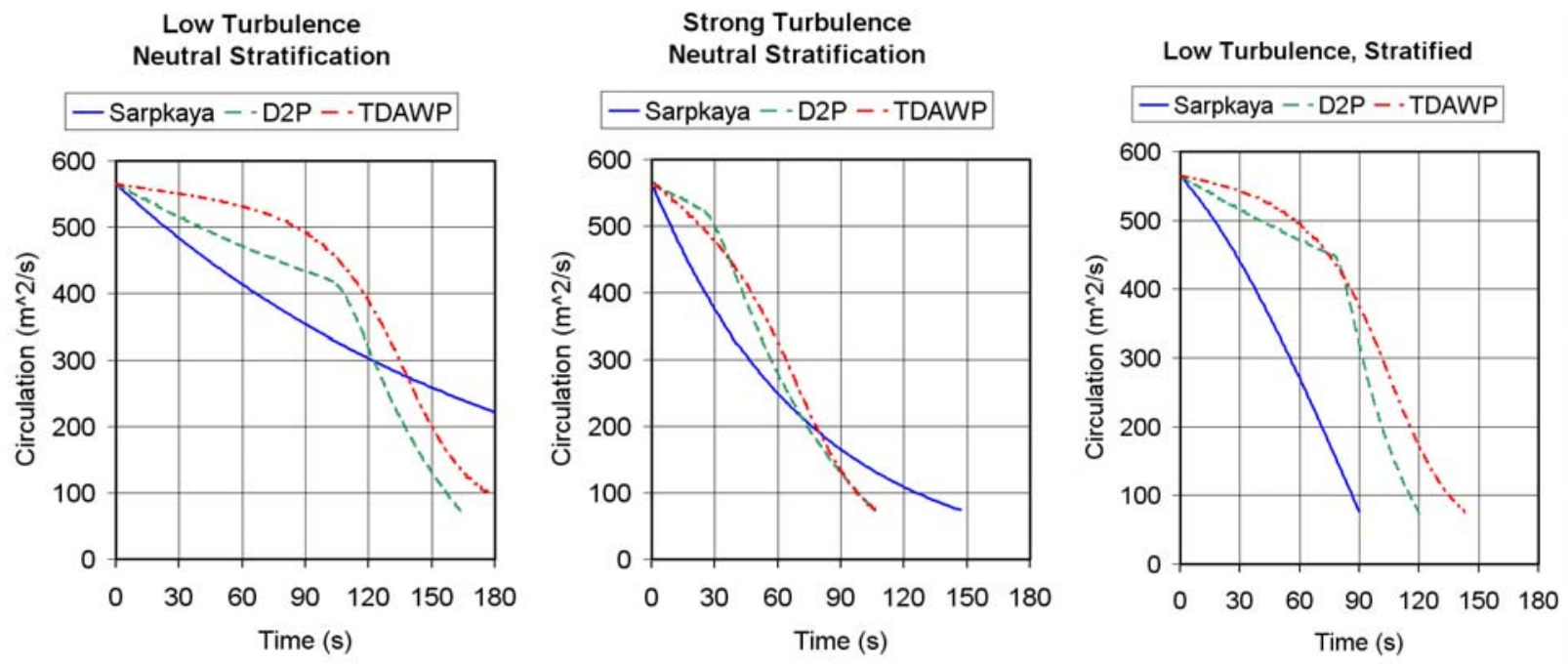

Figure 4. Circulation vs time comparison between OGE models.

American Institute of Aeronautics and Astronautics 092407 
LDV-Lidar, and a Monostatic Acoustic Velocimeter System (MAVS), as a B-757 flew at an altitude of $70 \mathrm{~m}$, up-wind of a well instrumented $200 \mathrm{ft}(61 \mathrm{~m})$ tower $^{32}$. Weather data with outstanding vertical resolution was obtained from the tower and tethersonde measurements ${ }^{33}$. The input sounding acquired from this data is shown in Fig. 5. The case was chosen as a benchmark due to the strong rebound from the crosswind shear by the downstream vortex ${ }^{34}$. As apparent in Fig. 6, observations and LES results using the TASS model shows that the downstream vortex rebounded upward as it descended into the zone that has a strong gradient of crosswind shear between 40-60 $m$ altitude (Fig. 5).

Predictions using APA 4.0 with Sarpkaya and TDAWP, are compared with Lidar and LES data in Fig. 6. Predictions from D2P are not included since it is expected to give results similar to Sarpkaya (both D2P

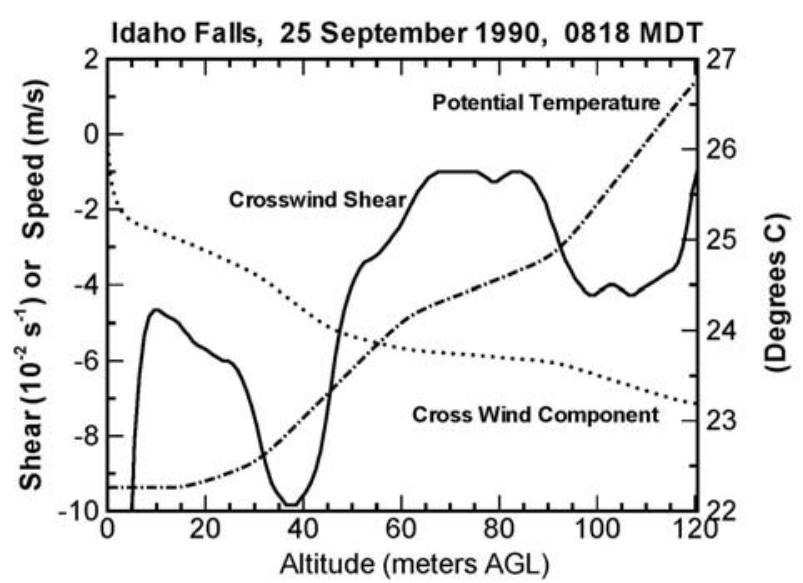

Figure 5. Input sounding for Idaho Falls Case. and Sarpkaya do not consider the effect of shear on altering the vortex descent rate). Figure. 6 shows that APA-Sarpkaya fails to predict the shear-induced rebound and underpredicts the transport of the downstream vortex since it remains in weaker crossflow near the ground. APATDAWP, on the other hand, predicts the downstream vortex to rebound with a similar trajectory as indicated by LES and measurements. Errors based on measured data at 60 seconds show the APA-TDAWP prediction of the downstream vortex to be $5 \mathrm{~m}$ too low, while the APA-Sarpkaya prediction to be $21 \mathrm{~m}$ too low. Lateral errors based on the downstream vortex at $60 \mathrm{~s}$ show the APA-TDAWP prediction to be within a couple of meters, while APASarpkaya has an error of $42 \mathrm{~m}$ ! Both APA-TDAWP and APA-Sarpkaya predict the upstream vortex to penetrate close to ground in good agreement with LES and measured data. The smaller error is because the upstream vortex is least affected by the crosswind shear.

\section{Statistical Evaluations}

In order to truly assess the overall strength and ranges of applicability of a given fast time model, a statistical evaluation using hundreds of field measurements with a large mix of aircraft should be used. Aircraft data should include type, weight, airspeed, altitude, and flight configuration. The first step should be to identify observed cases with data sets that include critical information such as aircraft data, weather information, and wake vortex
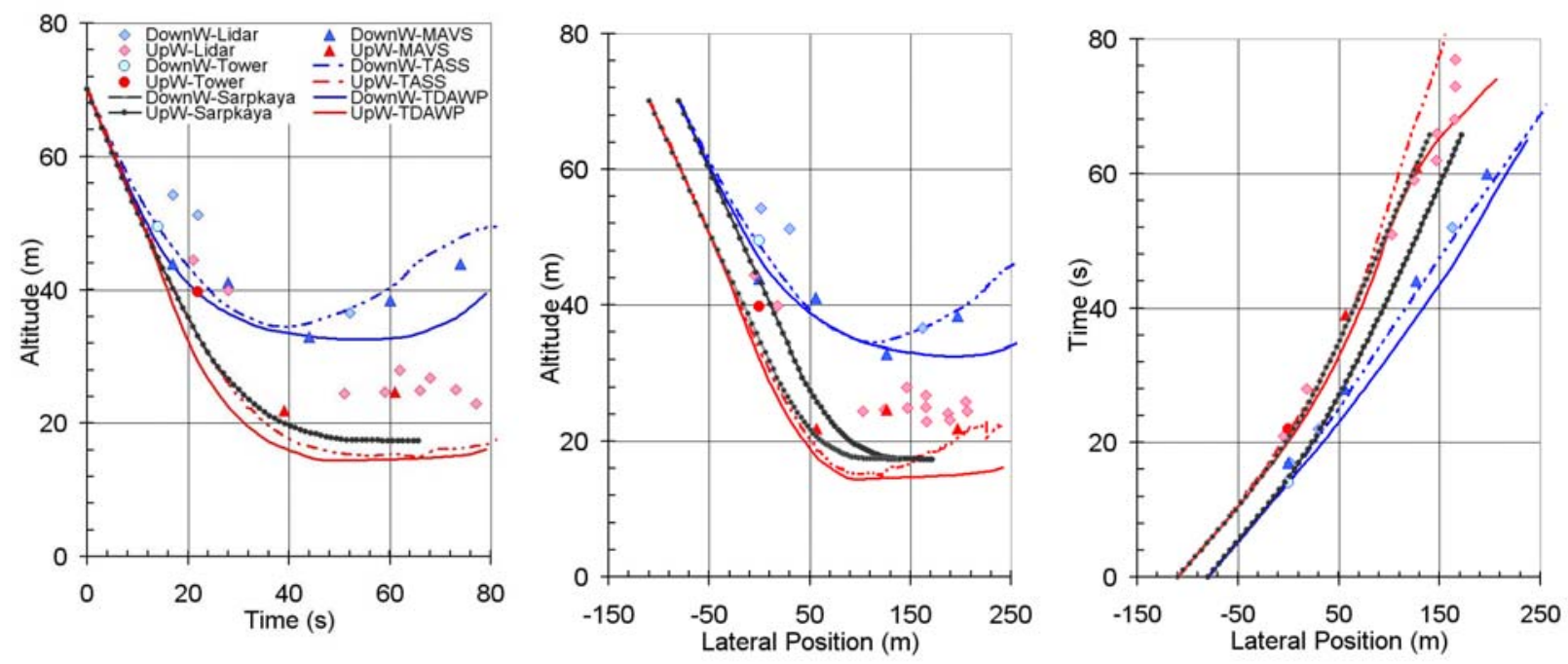

Figure 6. Idaho Falls run-9 case: comparison of trajectories between TASS-LES (dash dot), APA-Sarpkaya (black-dotted), and APA-TDAWP (solid) with observed data (symbols). Downwind vortex represented by blue and upwind red. 

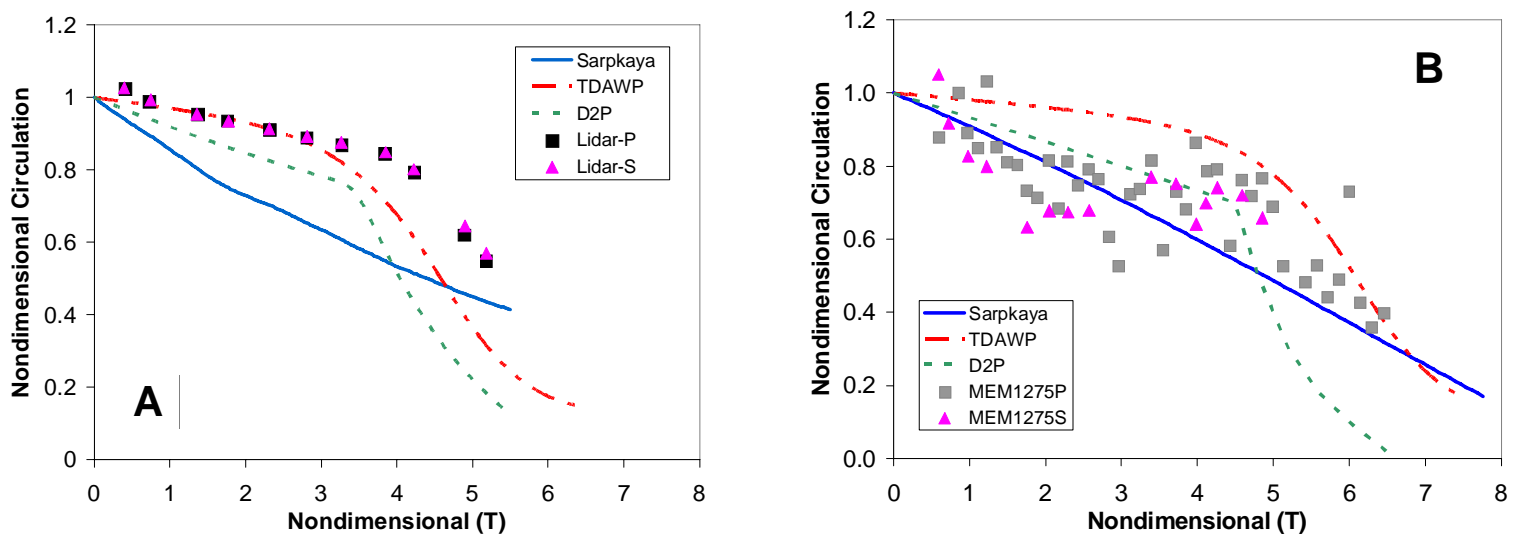

Figure 7. Example of model comparisons with Lidar in two unrelated OGE cases. Wake measurements in A) obtained by Pulsed Lidar and B) CW Lidar. Circulation for port vortex represented by squares, while starboard vortex represented by triangle.

measurements. This may require the visual inspection of the measured wake vortex strength and trajectory data, followed by the deselecting of inconsistent and incomplete data. Weather measurements include profiles of mean crosswinds, temperature and EDR. In some cases temperature data may be unavailable and temperature profiles for the time and location may need to be generated from mesoscale models. The quality of the wake vortex measurements and concurrent meteorological data depends upon the type of sensors, proximity to aircraft, temporal and spatial resolutions of the sensors, geography, and measurement strategy. Although numerous wake vortex field campaigns have occurred, very few measurement campaigns have been specifically designed to support evaluations for wake prediction models.

Statistical scoring of APA 3.1.1 with roughly 200 observed cases each from Memphis (MEM) and Dallas Ft-Worth (DFW) airports have been reported in Refs. 15 and 35. Root Mean Square (RMS) values were computed for each case based on differences between observed and predicted values of circulation, altitude, and lateral position. The approach in computing the RMS was to first interpolate both predictions and observations onto the same time grid. The square of the difference between the predicted and observed were computed for each time point and then averaged over the duration of the measurement. The scoring procedure was terminated when either the last Lidar datum or the predicted circulation goes to zero. The RMS values for circulation were normalized by its initial value while the RMS for altitude and lateral separation were normalized by the initial vortex separation. Mean RMS values and the $90^{\text {th }}$ percentile were reported separately for Memphis and DFW. Robins and Delisi also examined RMS statistics between the predicted and observed time of the wake to leave the flight corridor, via lateral or vertical transport. Differences for the statistics computed between MEM and DFW was probably due to the higher frequency of night time observations at MEM compared to DFW. Lower RMS errors for circulation were obtained for low-turbulence cases than for high turbulence.

Comparisons between D2P and APA-Sarpkaya using the above RMS approach also have been previously reported. Evaluations showed APA-Sarpkaya to score slightly better for circulation and lateral position, while D2P scored better for altitude, when scored with the MEM data set ${ }^{16}$. Subsequent evaluations using 64 cases measured with Pulsed Lidar in Germany indicated D2P to outscore APA-Sarpkaya, with significant differences for circulation $^{20}$. We have noticed that DLR's measurement's with Pulsed Lidar tends to produce higher and more consistent estimates of circulation than measurements with the MEM and DFW CW Lidars (see Fig. 7). This may be because the CW Lidar analysis produces circulation datum points that are underestimated due to poor focus, and may favor the APA-Sarpkaya's tendency for early rapid decay. Also, this is why it is important to understand and quantify the accuracy of data collected for model development and evaluations. Another contributing factor that may bias errors in favor of D2P is in the scoring procedure, which terminates with either the last Lidar measurement or when the predicted circulation goes to zero. Thus models (such as D2P) that underpredict the vortex lifetime, will not be scored against the later observed datum points. This could result in better scores for D2P, especially for altitude and lateral position, since uncertainties increase with time due to turbulence and other factors (e.g., see Lin, et $\left.\mathrm{al}^{36}\right)$. 

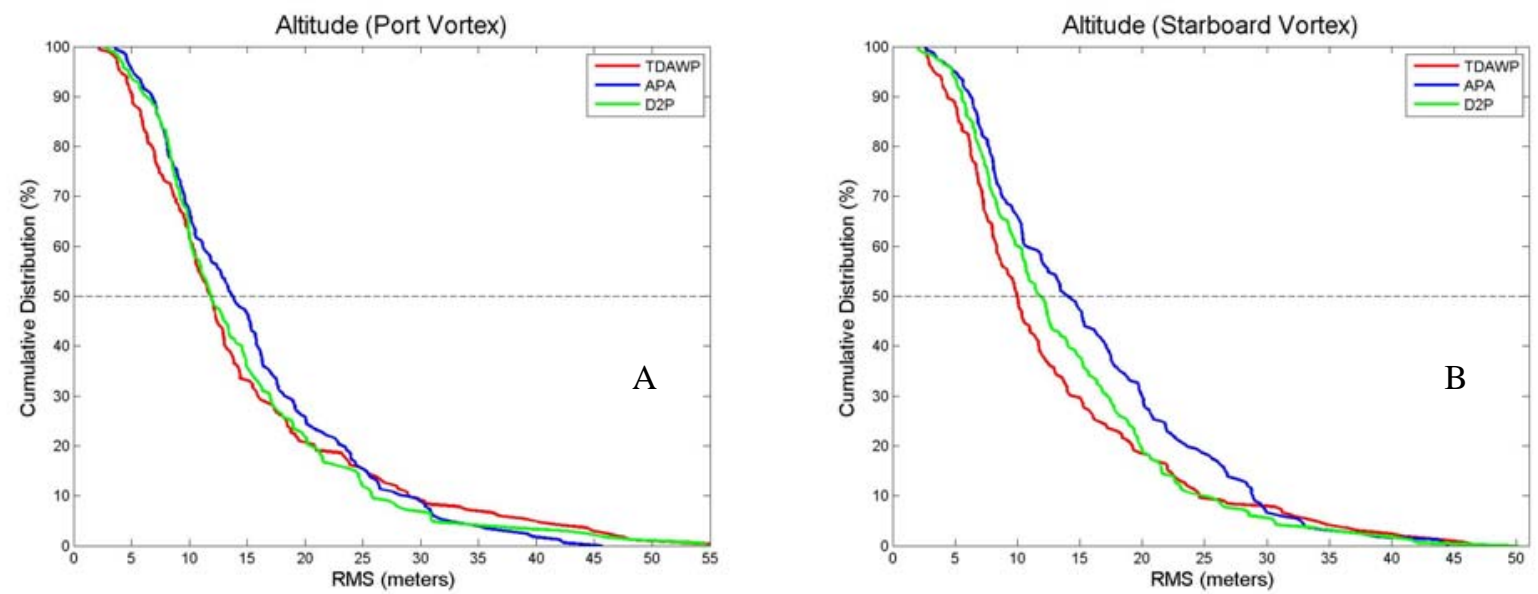

Figure 8. Cumulative distribution of RMS differences between Lidar and model predictions for wake vortex altitude for A) port and B) starboard vortex. Curves represent TDAWP (red), Sarpkaya (blue), and D2P (green).

\section{Statistical Evaluation with Denver Data}

Recent data from Denver International Airport (DIA) is used to statistically score the three OGE modules that are embedded within the APA Suite. Statistical analysis and scoring were adapted from software provided by NWRA. Due to the anticipated large number of cases, automation components were developed to 1) efficiently process and check data, 2) construct the atmospheric profile, 3) prepare initial conditions, 4) run the fast-time prediction models, and 5) statistically analyze differences between predictions and observations.

For the analysis, a specific 24 hour operational period for arrivals into DIA is selected. This period was chosen because of the availability of continuous Lidar measurements and coexisting meteorological data. The relative quality of the data over this period appeared very good. Another advantage is the analysis would not be biased by measurements taken during specific time of day. The full day of arrivals included 173 aircraft, ranging in aircraft types such as B-777 to CRJ7. The vast majority of the aircraft were Large-category aircraft such as the A-319 and B-737. A Pulsed Lidar scanned orthogonal to the approaching flight path of runway 16L. The average altitude of the flight path at this intersection was $228 \mathrm{~m}$ (750 ft), and was limited to viewing OGE cases.

Vertical profiles of crosswind, temperature, and EDR (needed for running the fast-time models) were obtained for each case. The crosswind profiles were taken from an average of Lidar-derived winds measured prior to, and after each aircraft. Profiles for EDR were computed from boundary layer similarity theory ${ }^{37}$ using the EDR derived
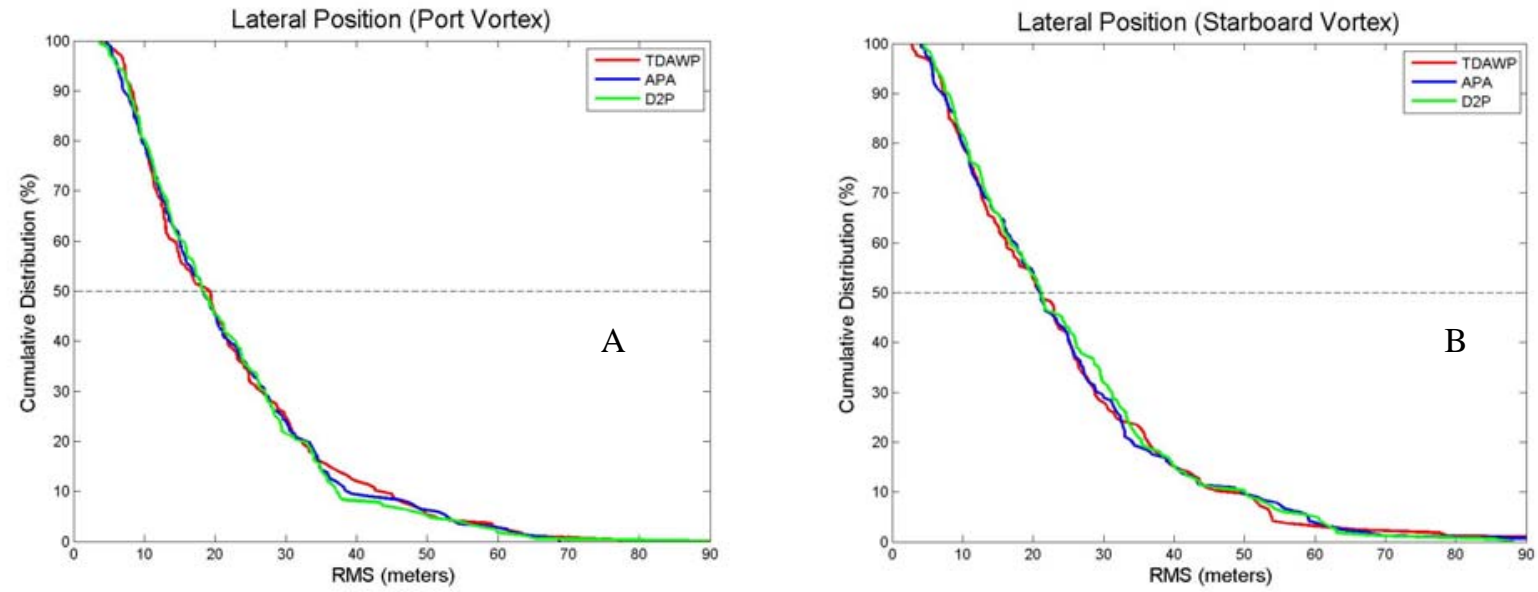

Figure 9. Cumulative distribution of RMS differences between Lidar and model predictions for wake vortex lateral position for A) port and B) starboard vortex. Curves represent TDAWP (red), Sarpkaya (blue), and D2P (green). 
from sonic anemometers on a nearby $106 \mathrm{ft}(32.3 \mathrm{~m})$ tower. Temperature profiles were obtained from short-term forecasts using a nested Mesoscale Weather Prediction (MM5) model. Fast-time model input for aircraft location was based on the detected aircraft position (rather than flight path). Elliptical loading was assumed for computing input initial circulation and vortex separation. Since the actual aircraft weights were not known, initial circulation was based on $90 \%$ of the maximum landing weight for each aircraft.

Scoring of the models is computed for lateral and vertical position as well as circulation. Only the results from the position data are presented due to the uncertainty in the quality of the measured circulations from this site. The distribution of RMS for altitude and lateral position are shown in Figs. 8 and 9 and summarized in Tables 1 and 2. The RMS data for lateral position is nearly equivalent between models. For altitude prediction of the starboard vortex, APA-TDAWP scores slightly better, and APA-D2P is next. However, all three models give nearly equivalent forecasts for the port vortex.

Table 1. Statistics for differences between model and Lidar observations for $24 \mathrm{hr}$ period at DIA (173 cases): Starboard vortex

\begin{tabular}{llll}
\hline \hline Model & Averages & RMS Lateral $(m)$ & RMS Vertical $(m)$ \\
\hline APA-Sarpkaya & Medium & 21 & 14 \\
& $90^{\text {th }}$ perc. & 49 & 29 \\
\hline APA-TDAWP & Medium & 21 & 10 \\
& $90^{\text {th }}$ perc. & 48 & 24 \\
\hline APA-D2P & Medium & 21 & 12 \\
& $90^{\text {th }}$ perc. & 50 & 24 \\
\hline \hline
\end{tabular}

The absolute RMS numbers are small due to the quality of crosswind measurements. A large contribution to the RMS is expected from wake meandering and sinusoidal instabilities (e.g., see Fig. 1) rather than from model inaccuracies. The sinusoidal meandering can contribute to errors in the ranges of about one wing span.

Future scoring of the models needs to address vortex lifetime biases in scoring approaches. In the current approach, position scoring was rewarded if the model underpredicts the lifetime of the vortex (relative to the other models). RMS differences with observed data were not computed once the model predicts the wake to have decayed. One possible way to correct for this is to keep the last predicted position, after the model has predicted the vortex to have no circulation.

Table 2. Statistics for differences between model and Lidar observations for $24 \mathrm{hr}$ period at DIA (173 cases): Port vortex

\begin{tabular}{llll}
\hline \hline Model & Averages & RMS Lateral $(\boldsymbol{m})$ & RMS Vertical $(\boldsymbol{m})$ \\
\hline APA-Sarpkaya & Medium & 19 & 14 \\
& $90^{\text {th }}$ perc. & 40 & 29 \\
\hline APA-TDAWP & Medium & 19 & 12 \\
& $90^{\text {th }}$ perc. & 42 & 29 \\
\hline APA-D2P & Medium & 19 & 12 \\
& $90^{\text {th }}$ perc. & 38 & 26 \\
\hline \hline
\end{tabular}

The RMS approach to scoring gives a measurement of how well the prediction is doing throughout the vortex lifetime. Other scoring approaches should be considered that give significant weight to the latter part of a history; i.e. at times when the potential for wake encounters exist from in-trail aircraft.

Also, future work needs to break down the scoring based on ranges of turbulence, stratification, and shear, in order to properly assess weaknesses and limitations of the model. An evaluation of Pulsed Lidar detection capabilities and a more extensive statistical evaluation of fast-time models are now being sponsored by NASA's Airportal Project.

\section{Conclusion}

Fast-time wake vortex models are currently being evaluated for possible operational and research applications. Deterministic fast-time wake predictions models compute histories of wake vortex position and strength, using 
aircraft and meteorological data as input. Deterministic fast-time models are recommended to form the basis for fasttime wake-zone and probabilistic models.

The deterministic fast-time models are scored using root-mean-squared differences between predictions and observations. For predictions of vortex trajectory, all of the evaluated OGE models seem to work well. The TDAWP model may have an advantage due to its capability to predict shear effect on vortex descent. Evaluations show that differences between observed histories and model predictions tend to grow with time, and thus it is important to compare errors from different models over similar time ranges Additional study is needed with more cases, especially for in ground effect.

It is interesting to see similarity in OGE wake predictions between TDAWP and D2P. This is surprising since the two models have very different formulations and their development was driven by parametric results from two different LES models. Both fast-time models predict two-phased circulation decay; however, the TDAWP predictions have slightly longer wake lifetimes. Only the TDAWP model has a formulation for crosswind shear effects on vortex descent rate.

The Sarpkaya model produces very different predictions for circulation than TDAWP or D2P, but may better represent the circulation data measured by CW Lidar to which it was tuned. However, comparisons with Pulsed Lidar favor TDAWP and D2P. An assessment of Lidar accuracy is needed to provide guidance as to which model is more accurate. The Sarpkaya model produces longer lived vortices than either TDAWP or D2P for near neutral stratification, but shorter lifetimes in stratified environments. Compared to the other two models, the Sarpkaya model tends to predict a faster weakening of vortex descent speed resulting in a smaller magnitude of descent (i.e.. the wake does not go down as far). These differences may not show up in the statistical scoring since they are small relative to other uncertainties, such as those due to wake meandering and sinusoidal instability.

Variability and errors from the sensing of meteorological data that is used as input could overwhelm the errors of the models. Improvements in sensors and the collection of weather data may lead to significant improvements in wake prediction. Future statistical evaluations of fast-time models need to address wake-lifetime biases in scoring approaches. Other fast-time models, if made available, could be added to future scoring evaluations.

\section{Acknowledgments}

We would like to acknowledge Bob Robins NWRA for his assistance with the fast-time and scoring software, Don Bagwell of NASA for his contribution in processing the Denver data, the FAA and Volpe Center for the collection and processing of field data, and technical contributions from Hazari Syed, of Raytheon Technical Services and George Switzer of Analytical Services \& Materials.

\section{References}

\footnotetext{
${ }^{1}$ Journade, J., “Numerical Wake Vortex Characterization,” $21^{\text {st }}$ Applied Aerodynamics Conference, 23-26 June 2003, AIAA 2003-3815.

${ }^{2}$ Holforty, W., and Powell, J.D., “Airborne Wake Avoidance and Visualization Experiment”, Annual International Symposium of the Society of Flight Test Engineers, 15-19 September 2003.

${ }^{3}$ Belocerkovsky, A., Falkov, E., and Kanevsky, M., "Russian Integrated Wake Vortex Flight Safety System,” FAA WakeNet USA, 21-22 October 2008, PowerPoint Presentation, 13 pp.

${ }^{4}$ Rouwhorst, W.F.J.A., Groeneweg, J., Winckelmans, G., and Luckner, R, "Piloted Simulation Results of an Onboard Wake Vortex Detection, Warning and Avoidance System and Cockpit Display Aspects," $26^{\text {th }}$ International Congress of the Aeronautical Sciences, 14-19 September 2008, ICAS-2008-9.9.1.

${ }^{5}$ Hinton, D.A., “Aircraft Vortex Spacing System (AVOSS) Conceptual Design,” August 1995, NASA TM-110184.

${ }^{6}$ Perry, R.B., Hinton, D.A., and Stuever, R.A., "NASA Wake Vortex Research for Aircraft Spacing," 35th Aerospace Sciences Meeting \& Exhibit, January 1997, AIAA 97-0057.

${ }^{7}$ O’Connor, C., and Rutishauser, D., "Enhanced Airport Capacity Through Safe, Dynamic Reductions in Aircraft Separation: NASA's Aircraft VOrtex Spacing System (AVOSS),” Journal of Air Traffic Control, Vol. 43, No. 3, October-December 2001, pp. 4-10.

${ }^{8}$ Rutishauser, D., Lohr, G., Hamilton, D., Powers, R., McKissick, B., Adams, C., and Norris, E., "Wake Vortex Advisory System (WakeVAS) Concept of Operations,” April, 2003, NASA TM 2003-212176.

${ }^{9}$ Bryant, W., Lang, S., Tittsworth, J., and Darr, J., "Wake Vortex Avoidance System," $1^{\text {st }}$ CEAS European Air and Space Conference, September 2007, CEAS-2007-175, pp. 3357-3369.

${ }^{10}$ Proctor, F.H., "The NASA-Langley Wake Vortex Modelling Effort in Support of an Operational Aircraft Spacing System," 36th Aerospace Sciences Meeting \& Exhibit, 12-15 January 1998, AIAA 98-0589.
} 
${ }^{11}$ Han, J., Lin, Y. -L., Schowalter, D. G., Arya, S. P., and Proctor, F. H., "Large Eddy Simulation of Aircraft Wake Vortices within Homogeneous Turbulence: Crow Instability,” AIAA Journal, Vol. 38, No. 2, Feb. 2000, pp. 292-300.

${ }^{12}$ Crow, S. C., "Stability Theory for a Pair of Trailing Vortices,” AIAA Journal, Vol. 8, No. 12, Dec. 1970, pp. $2172-2179$.

${ }^{13}$ Greene, G. C., “An Approximate Model of Vortex Decay in the Atmosphere,” Journal of Aircraft, Vol. 23, No. 7, 1986, pp. 566-573.

${ }^{14}$ Kantha, L.H., “Empirical Model of Transport and Decay of Wake Vortices Between Parallel Runways,” J. Aircraft, Vol. 33, No. 4, 1996, pp. 752-760.

${ }^{15}$ Sarpkaya, T., Robins, R.E., and Delisi, D.P., "Wake-Vortex Eddy-Dissipation Model Predictions Compared with Observations,” Journal of Aircraft, Vol. 38, No. 4, 2001, pp. 687- 692.

${ }^{16}$ Holzäpfel, F. and Robins, R.E., "Probabilistic Two-Phase Aircraft Wake-Vortex Model: Application and Assessment," Journal of Aircraft, Vol. 41, No. 5, 2004, pp. 1117-1126.

${ }^{17}$ Sarpkaya, T., "Final Report for The Creation of a Physics-based Ground-effect Model, Phase 2 - Inclusion of the Effects of Wind, Stratification, and Shear into the New Ground Effect Model, “ Contract No. NAS1-02117, NTRS ID: 20080013542, October 2006.

${ }^{18}$ Jackson, W., Yaras, M., Harvey, J., Winckelmas, G., Fournier, G., and Belotserkovsky, A., "Wake Vortex Prediction - An Overview”, Phase 6 and Project Final Report prepared for Transportation Development Centre and Transport Canada, TP 13629E, March 2001.

${ }^{19}$ Rossow, V.J. and Meyn, L.A., “Guidelines for Avoiding Vortex Wakes During Use of Closely-Spaced Parallel Runways,” $26^{\text {th }}$ AIAA Applied Aerodynamics Conference, 18-22 August 2008, AIAA-2008-6907.

${ }^{20}$ Holzäpfel, F., "Probabilistic Two-Phase Aircraft Wake-Vortex Model: Further Development and Assessment,” Journal of Aircraft, Vol. 43, No. 3, 2006, pp. 700-708.

${ }^{21}$ Stull, R.B., An Introduction to Boundary Layer Meteorology, Kluwer Academic Publishers, Dordrecht, 1988, 666 pp.

${ }^{22}$ Robins, R.E., and Delisi, D.P., “NWRA AVOSS Wake Vortex Prediction Algorithm Version 3.1.1,” June 2002, NASA CR2002-211746.

${ }^{23}$ Sarpkaya, T., “New Model for Vortex Decay in the Atmosphere,” Journal of Aircraft, Vol. 37, No. 1, 2000 , pp. 53-61.

${ }^{24}$ Proctor, F.H., Hamilton, D.W., and Switzer, G.F. "TASS Driven Algorithms for Wake Prediction," 44th Aerospace Sciences Meeting \& Exhibit, 9-12 January 2006, AIAA 2006-1073.

${ }^{25}$ Holzäpfel, F., "Probabilistic Two-Phase Wake Vortex Decay and Transport Model,” Journal of Aircraft, Vol. 40, No. 2, March-April, 2003, pp. 323-331.

${ }^{26}$ Proctor, F.H., Hamilton, D.W. and Han, J., "Wake Vortex Transport and Decay in Ground Effect: Vortex Linking with the Ground,” 38th Aerospace Sciences Meeting \& Exhibit, 10-13 January 2000, AIAA-2000-0757.

${ }^{27}$ Campbell, S.D., Dasey, T.J., Freehart, R.E., Heinrichs, R.M., Matthews, M.P., Perras, G.H., and Rowe, G.S., "Wake Vortex Field Measurement Program at Memphis, TN Data Guide,” April 1997, NASA CR-201690.

${ }^{28}$ Dasey, T.J., Cole, R.E., Heinrichs, R.M., Matthews, M.P., and Perras, G.H., “Aircraft Vortex Spacing System (AVOSS) Initial 1997 System Deployment at Dallas/Ft. Worth (DFW) Airport,” 8 July 1998, Project Report NASA/A-1; PB99-175598.

${ }^{29}$ Holzäpfel, F., Gerz, T., and Baumann, R., "The Turbulent Decay of Trailing Vortex Pairs in Stably Stratified Environments,” Aerospace Science and Technology, Vol. 5, No. 2, 2001, pp. 95-108.

${ }^{30}$ Proctor, F.H., and Switzer, G.F., "Numerical Simulation of Aircraft Trailing Vortices," 9th Conf. on Aviation, Range and Aerospace Meteorology, American Meteorology Society, 11-15 Sept 2000, pp. 511-516.

${ }^{31}$ Switzer, G.F., and Proctor, F.H., "Numerical Study of Wake Vortex Behavior in Turbulent Domains with Ambient Stratification, “38th Aerospace Sciences Meeting \& Exhibit, 10-13 January 2000,AIAA-2000-0755.

${ }^{32}$ Garodz, L.J., and Clawson, K.L., "Vortex Wake Characteristics of B757-200 and B767-200 Aircraft Using the Tower FlyBy Technique, Volumes 1 and 2," January 1993, NOAA Tech. Memo. ERL ARL-199.

${ }^{33}$ Systems Research Corporation, "Atmospheric Description for Idaho Falls B-757 Run \#9 on September 25, 1990," Volpe National Transportation Systems, Cambridge, MA, Jan. 1994.

${ }^{34}$ Proctor, F.H., Hinton, D.A., Han, J., Schowalter, D.G., and Lin Y.-L., "Two Dimensional Wake Vortex Simulations in the Atmosphere: Preliminary Sensitivity Studies,” 35th Aerospace Sciences Meeting \& Exhibit, January 1997, AIAA 97-0056.

${ }^{35}$ Robins, R.E., and Delisi, D.P., “Wake Vortex Algorithm Scoring Results,” June 2002, NASA CR-2002-211745.

${ }^{36}$ Lin, Y-L, Han, J., Zhang, J., Ding, F., Arya, S.P., and Proctor, F.H., "Large Eddy Simulation of Wake Vortices in the Convective Boundary Layer,” 38th Aerospace Sciences Meeting \& Exhibit, 10-13 January 2000, AIAA-2000-0753.

${ }^{37}$ Han, J.; Arya, S.P.; Shaohua, S.; Lin, Y-L., “An Estimation of Turbulent Kinetic Energy and Energy Dissipation Rate Based on Atmospheric Boundary Layer Similarity Theory,” June 2000, NASA CR-2000-210298. 